\title{
Three-Dimensional Measurement System Using a Cylindrical Mirror
}

\author{
Yuuki Uranishi, Mika Naganawa, Yoshihiro Yasumuro, Masataka Imura, \\ Yoshitsugu Manabe, and Kunihiro Chihara \\ Graduate School of Information Science, Nara Institute of Science and Technology, \\ 8916-5 Takayama Ikoma, Nara, Japan \\ \{yuuki-u, naganawa, yasumuro, imura, manabe, chihara\}@is.naist.jp \\ http://chihara.naist.jp/
}

\begin{abstract}
We propose a novel method for measuring a whole threedimensional shape of an object with a simple structured system. The proposed system consists of a single CCD camera and a cylindrical mirror. A target object is placed inside the cylindrical mirror and an image is captured by the camera from above. There are two or more points that have the same origin in the captured image: one is observed directly, and the other is observed via the mirror. This situation means that a point is observed by a real camera and virtual cameras at the same time. Therefore, the three-dimensional shape of the object can be obtained using stereo vision. We simulated an actual experimental situation and measured the three-dimensional shape of the object from the simulated image, and the results have demonstrated that the proposed method is useful for measuring the whole three-dimensional shape.
\end{abstract}

\section{Introduction}

Whole three-dimensional shape measuring and model reconstructing have a wide area of applications, including virtual museums [1] and digital archiving of relics [2. Most of whole three-dimensional models are built from multiple range images. Registration and integration of the images are cumbersome procedures. Therefore, it is highly desirable that a whole shape of the object is easily measured in a single shot. The three-dimensional measurement methods are classified into two groups: one is an active measurement method, and the other is a passive measurement method.

The active measurement method, such as Time-of-Flight [3] and Structured Lighting [4, obtains a shape of the target object by projecting a light or some kind of energy and by measuring its reflection. This method provides a precise shape of the object as a dense point cloud. However, it is impossible to obtain the whole shape data at once due to the limitation of the viewpoint. Therefore, in order to reconstruct the whole shape data, measurement from multiple viewpoints is required by moving a turntable or the sensor itself.

There are several existing methods for passive measurement. For example, Visual Hull [5] 6] constructs approximation of a whole object shape from silhou- 
ettes observed from multiple viewpoints using a volume intersection technique. This method is fast and robust enough to implement real-time applications. Although increasing the number of cameras improves the accuracy of an approximation of the actual object shape, a complete multi-camera calibration becomes inevitable. The accurate three-dimensional shape of the object can be measured using stereo vision [7], which is one of the passive methods. The geometric structure is acquired from images shoot from different viewpoints based on triangulation. It is difficult to force all cameras to be calibrated and synchronized. To overcome the problem, a catadioptric stereo system is proposed [8] 9]. The catadioptric stereo system consists of a single camera and one or more mirrors. Placing mirrors produces multiview observations that are included in a single shot image. There is the one that comes directly into the lens and there are those that are reflected by the mirrors. Conventional catadioptric stereo system involves a complex arrangement of mirrors and the object. Therefore, it becomes more difficult to arrange the system appropriately for whole shape measuring.

In this paper, we propose a simple structured method for measuring a whole three-dimensional shape with a single CCD camera and a cylindrical mirror. We have previously proposed a concept of our system [10]. An image of the target object, which is placed inside the cylindrical mirror, is captured from above. The captured image includes sets of points observed from multiple viewpoints. The three-dimensional shape of the object can be obtained using stereo vision. The proposed method is suitable for measuring the shape of moving objects such as insects and small animals, because the proposed method can measure a whole three-dimensional shape of the object in a single shot. In this study, we demonstrate the proposed method in a simulated experiment using a circular cone as a target object.

\section{Proposed System}

\section{$2.1 \quad$ System Overview}

The proposed system consists of a CCD camera to which the fish-eye lens is attached and a cylindrical mirror whose inside is coated by a silver reflective layer. Figures 1 (a) and (b) show a bird-eye view and a side view of the proposed system, respectively. A target object is placed in the cylindrical mirror, and an image is captured by the CCD camera from right above. As shown in Fig. 1 (b), each point of the object can be measured from different directions: one is a direct observation, and the others are the observations via the mirror. This situation implies that a given point is observed by a real camera and virtual cameras at the same time.

\subsection{Captured Image}

Figures 2(a) and (b) simulate the captured images by the proposed system. The object is set on the center of the cylindrical mirror in Fig. 2 (a) and off the center 


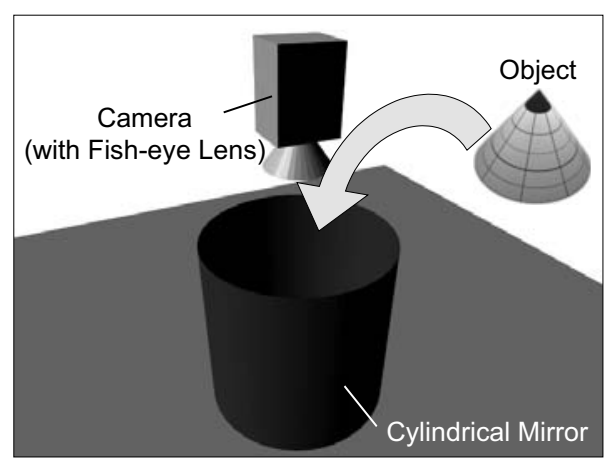

(a)

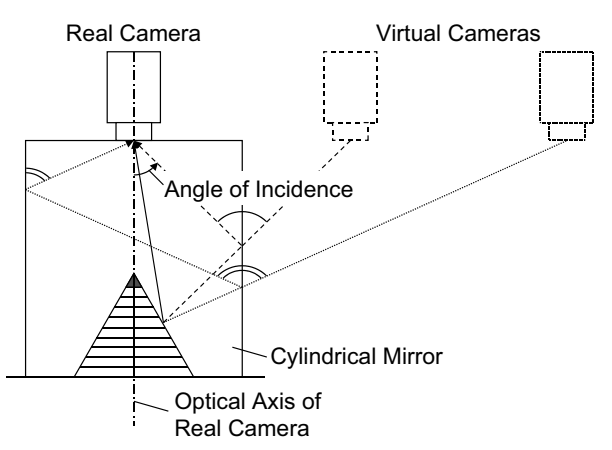

(b)

Fig. 1. A schematic overview of the proposed system. (a) The system consists of a CCD camera with fish-eye lens and a cylindrical mirror. A target object is placed inside the cylindrical mirror, and an image is captured by CCD camera from above. (b) Each point of the object can be observed directly and via the mirror. This situation implies that a point is observed by a real camera and virtual cameras in a single shot

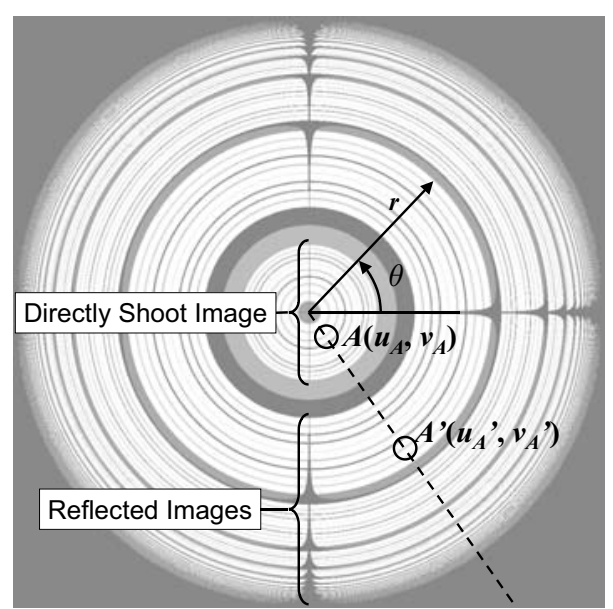

(a)

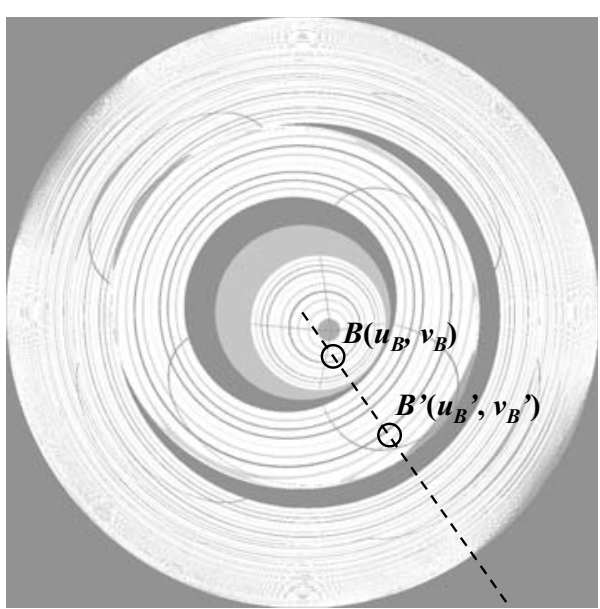

(b)

Fig. 2. The images captured by the proposed system in case of setting a circular cone on the center of the mirror (a) and off the center of the mirror (b). The area around the center of the image is a directly shoot image, and the peripheral area is a reflected image on the cylindrical mirror. When the optical axis of the camera is identical with the center axis of the mirror, the original point $A(B)$ and the reflected point $A^{\prime}\left(B^{\prime}\right)$ are always placed on the same line through the center of the cylindrical mirror

in Fig. 2 (b). The area around the center of the image is a directly shoot image, and the other area is a reflected image on the cylindrical mirror. When the optical 
axis of the camera is identical with the center axis of the mirror, the original point $A\left(u_{A}, v_{A}\right)\left(B\left(u_{B}, v_{B}\right)\right)$ and the reflected point $A^{\prime}\left(u_{A}^{\prime}, v_{A}^{\prime}\right)\left(B^{\prime}\left(u_{B}^{\prime}, v_{B}^{\prime}\right)\right)$ always lie on the same line through the center of the cylindrical mirror, which does not depend on the position of the object. Consequently, the search area for corresponding points is reduced to a line. The proposed system has a small calculation cost and yields small mismatches between the original points and the reflected points.

\subsection{Searching Stereo Pair}

The set of corresponding points that have same origin should be estimated to measure three-dimensional shape. Sum of Square Difference (SSD) is employed in this system. First, the captured images are represented in a polar coordinate system. Next, the area of the windows for SSD is normalized because the size of the window is changed dynamically according to its position in the image. Then, the set of points are searched using SSD. The detail of each step is described below.

Coordinate Conversion into Polar Coordinate System. A coordinate system of the captured image is converted into a polar coodinate system to facilitate calculating SSD values. Figure 3 is the image converted from Fig. 2 (a). The vertical and the horizontal axes in Fig. 3 correspond to a radius $r$ and an angle $\theta$ in Fig. 2 (a), respectively.

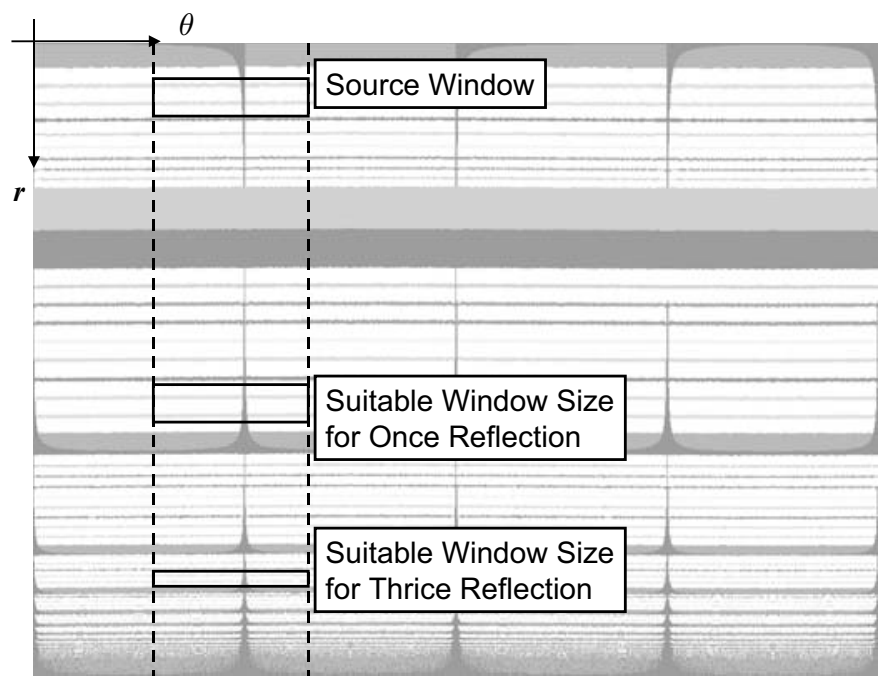

Fig. 3. The captured image Fig. 2 (a) in a polar coordinate system. The coordinate system of the captured image is converted into the polar coordinate system. The vertical and the horizontal axes in the converted image correspond to the radius $r$ and the angle $\theta$ in the original image, respectively 
Stretching the Target Region. The vertical length of the reflected image depends on the angle of incidence and a normal direction of the surface. As shown in Fig. 3, the vertical length changes according to the number of reflection. For example, the vertical length of the thrice reflected image is smaller than that of the once reflected image in Fig. 3. The optimal choice of the window size for SSD cannot be derived analytically, because the normal direction at each point of the object is unknown. Therefore, the combinations of the various window size are prepared and examined. The selected regions should have a same size for the calculation of SSD values. The target region is stretched to the same size as the source region. A converted luminance of $\mathrm{R}, \mathrm{G}$, and $\mathrm{B}, l_{i}(u, v)(i=r, g, b)$, is represented as follows:

$$
\begin{aligned}
v_{\text {int }} & \equiv \operatorname{trunc}\left(\frac{v}{s}\right), \\
\Delta & \equiv \frac{v}{s}-v_{\text {int }}, \\
l_{i}(u, v) & =(1-\Delta) o_{i}\left(u, v_{\text {int }}\right)+\Delta o_{i}\left(u, v_{\text {int }}+1\right) \quad(i=r, g, b),
\end{aligned}
$$

where $s$ is a ratio of the vertical length of the target region to that of the source region, and $o_{i}(i=r, g, b)$ is the original luminance of each color at $(u, v)$, and trunc is a function that truncates a number to an integer.

Calculating SSD. SSD value $d_{S T}$ between the source region $S$ and the target region $T$ is described as

$$
\begin{aligned}
l l d_{S T}(u, v)= & \sum_{u, v}\left\{\left(l_{r}^{S}(u, v)-l_{r}^{T}(u, v)\right)^{2}+\left(l_{g}^{S}(u, v)-l_{g}^{T}(u, v)\right)^{2}\right. \\
& \left.+\left(l_{b}^{S}(u, v)-l_{b}^{T}(u, v)\right)^{2}\right\}
\end{aligned}
$$

where $l_{i}^{S}(u, v)$ and $l_{i}^{T}(u, v)(i=r, g, b)$ are luminance of color at the source region and the target region, respectively. The set of points that provides the smallest SSD value is selected as an optimal set.

\subsection{Estimating the Position of the Virtual Cameras}

The position of the virtual cameras is estimated from the captured image. Figure 4 shows a relationship between an angle from an optical axis of the lens and a distance from a center of the captured image. In the proposed system, the distance from the center of the image $d$ is defined as

$$
d=f \theta_{o},
$$

where $f$ is a focal length of the lens and $\theta_{o}$ is an angle from the optical axis of the lens.

A position of a virtual camera is determined by the angle $\theta$, a radius of the cylindrical mirror and a number of reflection. Figure 5 shows how a camera position is determined. Assuming that the position of a real camera is the point 


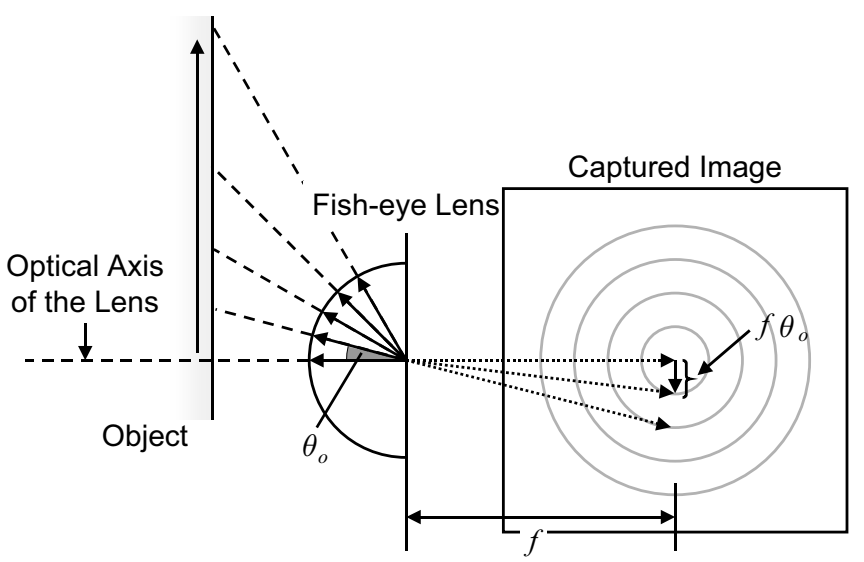

Fig. 4. A relationship between an angle from an optical axis of the lens and a distance from a center of the image. The distance from the center of the image is proportional to the angle from the optical axis. Such a projection is called as an equidistance projection

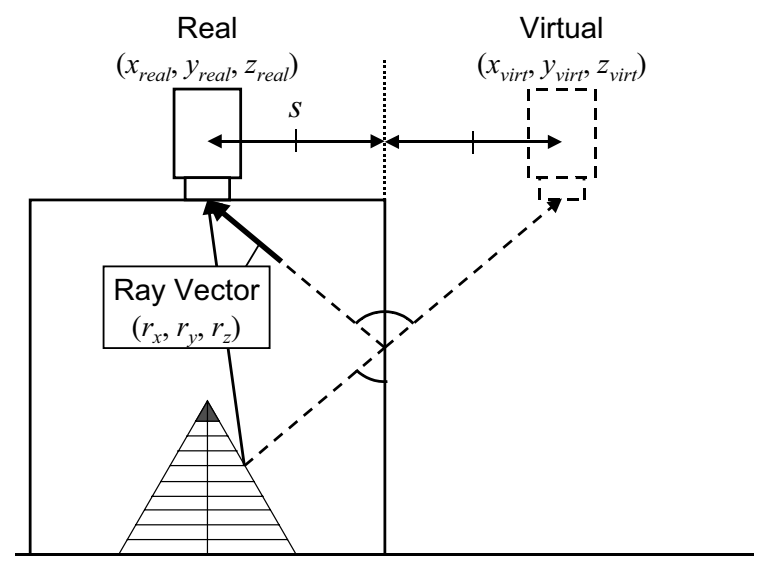

Fig. 5. Estimation of the position of the virtual camera. A position of a virtual camera can be estimated using Eqs. (6), (7) and (8)

$C_{\text {real }}$ at $\left(0, y_{\text {real }}, 0\right)$ and the height of the virtual camera is same as that of the real camera, the position of a virtual camera that corresponds to the $n$th reflection is represented as

$$
\begin{aligned}
& x_{\mathrm{virt}}^{n}=(-1)^{2 n-1}(2 n R \cos \theta), \\
& y_{\mathrm{virt}}^{n}=y_{\text {real }}, \\
& z_{\mathrm{virt}}^{n}=(-1)^{2 n-1}(2 n R \sin \theta),
\end{aligned}
$$

where $R$ is a radius of a cylindrical mirror. 


\section{$3 \quad$ Experimental Results}

An actual experimental situation was simulated and the three-dimensional shape of the target object was estimated from the simulated image. A simulation environment is shown in Fig. 6. A target object, a circular cone, was placed inside the cylindrical mirror. The camera was set at the same altitude as the top of the cylinder, and the optical axis of the camera was arranged to be coincident with the optical axis of the cylindrical mirror. The diameter and the height of the cylinder are $102.4 \mathrm{~mm}$, and the cone bottom diameter and the cone height are $76.8 \mathrm{~mm}$ and $38.4 \mathrm{~mm}$, respectively. The cone has differently colored lines at even intervals. A profile of the fish-eye lens is assumed to be an equidistance projection. The size of the captured image is $1024 \times 1024$ pixels, and the size of the converted image in the polar coordinate system is also $1024 \times 1024$ pixels.

Figure 7 (a) shows a reconstructed model from Fig. 2 (a) using SSD with a $20 \times 15$ size window, and Fig. 7 (b) is the actual shape of the target cone. The source region for calculating the value of SSD is limited to the curves that are drawn on the surface of the cone. The value of $s$ in Eq. (1) varies from 0.6 to 1.0 in steps of 0.1 . In addition, only the directly shoot image and the once reflected image are used to reconstruct this model. As shown in Fig. [7 the shape of the obtained model was almost similar to the actual shape of the cone. However, the bottom of the cone failed to be measured appropriately.

Figure 8 shows the case of identifying the set of points to measure the cone bottom. The true corresponding regions are the regions $S$ and $T_{1}$. However, the region $T_{2}$ was estimated instead of $T_{1}$ in the simulation. This is because the curve's color in the region $T_{2}$ is similar to that in the region $S$. The SSD

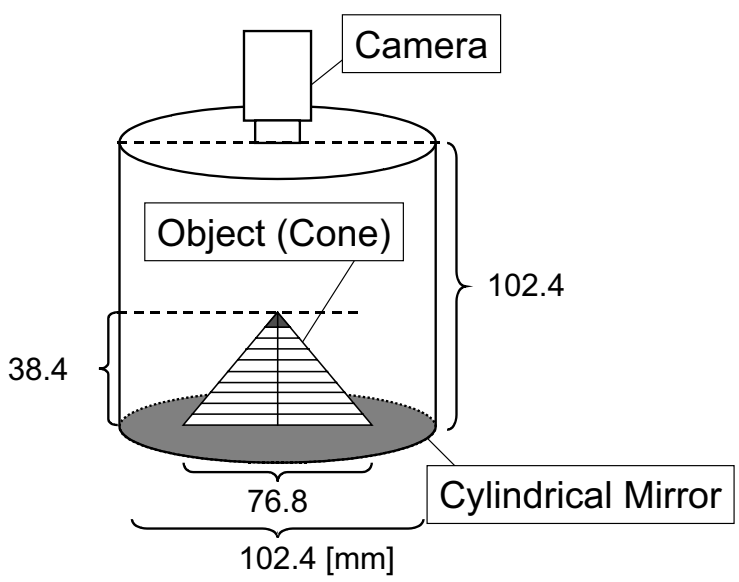

Fig. 6. An illustration of a simulation environment. A circular cone, which is a target object, is placed inside the cylindrical mirror. The optical axis of the camera is coincident with the center axis of the cylindrical mirror. The altitude of the camera lens is same as the top of the cylinder 


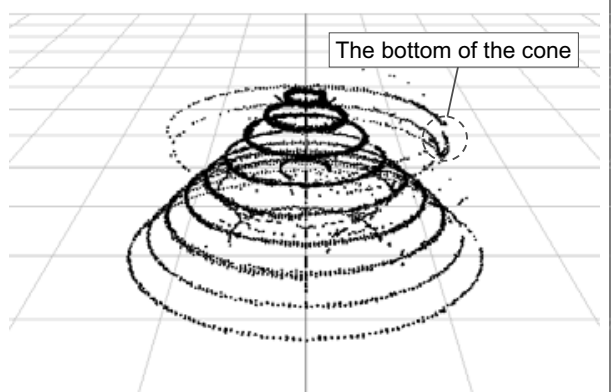

(a)

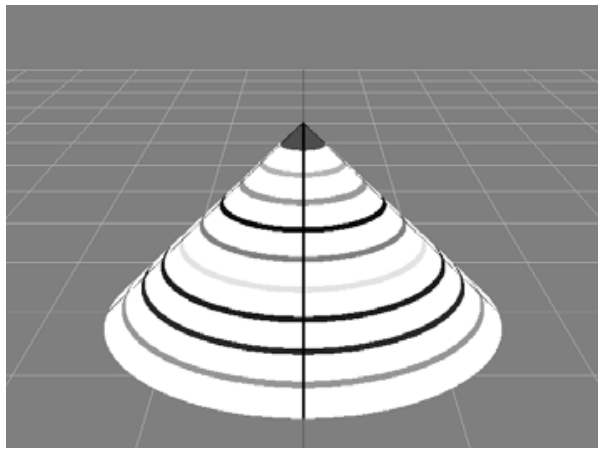

(b)

Fig. 7. Reconstructed three-dimensional model from Fig. 2 (a) using the proposed method (a) and the actual shape of the target cone (b). These figures show that the shape of the obtained model using the proposed method is similar to the actual shape of the cone

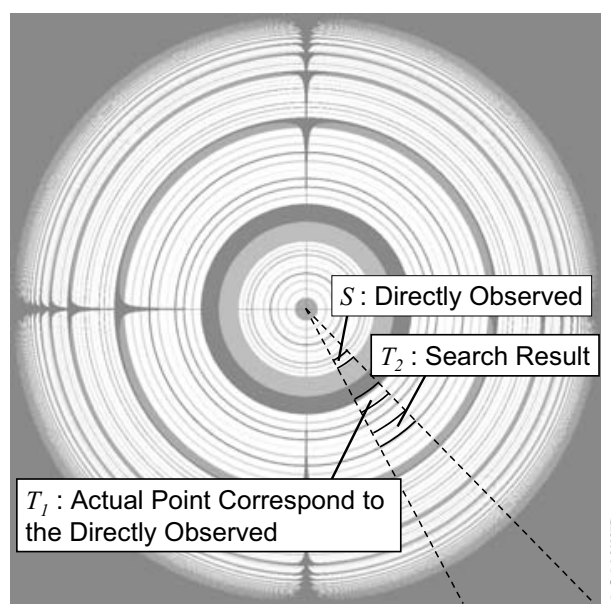

(a)

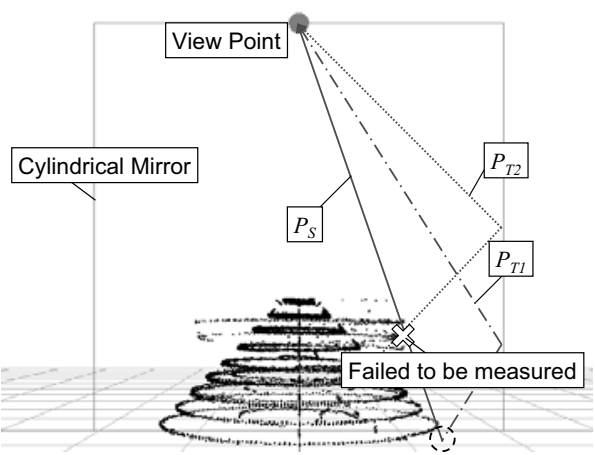

(b)

Fig. 8. An example of searching the set of points at the bottom of the cone. (a) The regions in the captured image. $S$ is the directly observed region, $T_{1}$ is the actual region that corresponds to the region $S$, and $T_{2}$ is the region that has the smallest SSD value between the region $S$. (b) A side view of the reconstructed model. The paths $P_{S}, P_{T 1}$ and $P_{T 2}$ correspond to the region $S, T_{1}$ and $T_{2}$ in the figure (a), respectively

value $d_{S T 2}$ between the regions $T_{2}$ and $S$ was smaller than that $d_{S T 1}$ between the regions $T_{1}$ and $S$. Therefore, the height of the cone bottom failed to be estimated using the path $P_{S}$ and the incorrect path $P_{T 2}$. To avoid these mismatches, the outlier points are diminished by resizing the optimal window based on the reconstructed model. 
In addition, some error factors should be taken into consideration in case of implementing the system. The cylindrical mirror in the actual system have distortions. Furthermore, it is difficult that the optical axis of the camera is set to be precisely identical with the center axis of the mirror. These error factors cause mismatches in searching the stereo pairs due to the geometrical distortion of the image. Distortion correction using a calibration pattern may be beneficial to search the correct stereo pairs.

\section{Conclusion}

In this paper, we have proposed the method for the three-dimensional measurement using a CCD camera and a cylindrical mirror. The three-dimensional shape of the object can be easily measured in a single shot using the proposed method. The result demonstrated that the similar three-dimensional model to the actual shape was reconstructed from the captured image. However, the SSD value between images that have a similar color was smaller than the value between the actual pair, and the shape of the target object failed to be measured in some cases.

Future work will aim at developing a robust method for searching the set of points in case that the several candidate regions with similar color or texture exist in the captured image. In addition, we are planning to build a prototype system and measure the three-dimensional shape of the real object using the prototype system.

\section{Acknowledgement}

This research is partly supported by Core Research for Evolutional Science and Technology (CREST) Program "Advanced Media Technology for Everyday Living" of Japan Science and Technology Agency (JST).

\section{References}

1. Shiaw, H., Jacob, R.J.K., Crane, G.R.: The 3D vase museum: A new approach to context in a digital library. Proceedings of the 4th ACM/IEEE-CS Joint Conference on Digital Libraries (2004) 125-134

2. Levoy, M., Pulli, K., Curless, B., Rusinkiewicz, S., Koller, D., Pereira, L., Ginzton, M., Anderson, S., Davis, J., Ginsberg, J., Shade, J., Fulk, D.: The digital michelangelo project: 3D scanning of large statues. Proceedings of ACM SIGGRAPH 2000 (2000) 131-144

3. Ullrich, A., Studnicka, N., Riegl, J.: Long-range high-performance time-of-flightbased 3D imaging sensors. Proceedings of the First International Symposium on 3D Data Processing Visualization and Transmission (2002) 852-855

4. Sato, K., Inokuchi, S.: Three-dimensional surface measurement by space encoding range imaging. Journal of Robotic Systems 2 (1985) 27-39 
5. Laurentini, A.: The visual hull concept for silhouette-based image understanding. IEEE Transactions on Pattern Analysis and Machine Intelligence 16 (1994) 150162

6. Matusik, W., Buehler, C., Raskar, R., Gortler, S.J., McMillan, L.: Image-based visual hulls. Proceedings of ACM SIGGRAPH 2000 (2000) 369-374

7. Klette, R., Schlüns, K., Koschan, A.: Computer Vision: Three-Dimensional Data from Images. Springer-Verlag Singapore (1998)

8. Gluckman, J., Nayar, S.K.: Rectified catadioptric stereo sensors. Proceedings of IEEE Conference on Computer Vision and Pattern Recognition (2000) 2380-2387

9. Zhang, Z.Y., Tsui, H.T.: 3D reconstruction from a single view of an object and its image in a plane mirror. Proceedings of International Conference on Pattern Recognition 2 (1998) 1174-1176

10. Manabe, Y., Uranishi, Y., Yasumuro, Y., Imura, M., Chihara, K.: Threedimensional measurement for small moving object. Proceedings of SPIE-IS\&T Electronic Imaging 5665 (2005) 235-242 